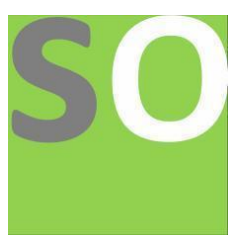

Article title: The Nicholson's conjecture

Authors: Jan Feliksiak[1]

Affiliations: N. A.[1]

Orcid ids: $0000-0002-9388-1470[1]$

Contact e-mail: jan.feliksiak1@yahoo.com

License information: This work has been published open access under Creative Commons Attribution License http://creativecommons.org/licenses/by/4.0/, which permits unrestricted use, distribution, and reproduction in any medium, provided the original work is properly cited. Conditions, terms of use and publishing policy can be found at https://www.scienceopen.com/.

Preprint statement: This article is a preprint and has not been peer-reviewed, under consideration and submitted to ScienceOpen Preprints for open peer review.

DOI: 10.14293/S2199-1006.1.SOR-.PPT1JIB.v1

Preprint first posted online: 24 February 2021

Keywords: Distribution of primes, Prime gaps bound, Maximal prime gaps bound, Nicholson's conjecture, Prime number theorem 


\title{
THE NICHOLSON'S CONJECTURE
}

\author{
JAN FELIKSIAK
}

ABstract.

This research paper discusses the distribution of the prime numbers, from the point of view of the Nicholson's Conjecture of 2013:

$$
\left(\frac{p_{(n+1)}}{p_{(n)}}\right)^{n} \leq n \log n
$$

The proof of the conjecture permits to develop and establish a Supremum bound on:

$$
\log n-\frac{1}{n}\left(\frac{p_{(n+1)}}{p_{(n)}}\right)^{n} \leq \mathcal{S U P}
$$

Nicholson's Conjecture belongs to the class of the strongest bounds on maximal prime gaps.

2000 Mathematics Subject Classification. 0102, 11A41, 11K65, 11L20, 11N05, 11N37,1102, 1103.

Key words and phrases. Distribution of primes, maximal prime gaps upper bound, Nicholson's conjecture, Prime Number Theorem. 


\section{Preliminaries}

Within the scope of the paper, prime gap of the size $\mathfrak{g} \in \mathbb{N} \mid \mathfrak{g} \geq 2$ is defined as an interval between two primes $p_{n}, p_{(n+1)}$, containing $(\mathfrak{g}-1)$ composite integers. Maximal prime gap of the size $\mathfrak{g}$, is a gap strictly exceeding in size any preceding gap. All calculations and graphing were carried out with the aid of the Mathematica ${ }^{\circledR}$ software. For all $n \in \mathbb{N} \mid n \geq 8$, we make the following definitions:

Definition 1.1 (Maximal Prime Gap).

$$
\mathfrak{g}=p_{(n+1)}-p_{n}
$$

A variant of logarithmic scaling of the horizontal axis is given by:

Definition 1.2 (Scaling factor). $\xi=\frac{\log _{10}\left(\frac{n}{24}\right)}{\log _{10}(24)}$
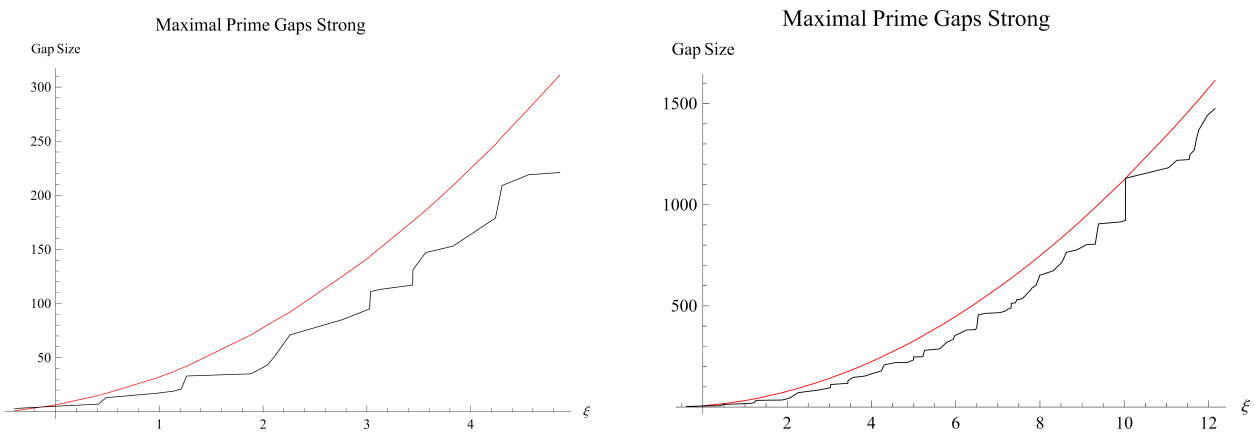

FIGURE 1. shows the graphs of $\mathcal{G}_{(n)}$ (red) and the actual maximal gaps (black) with respect to $\xi$ as given by Def. 1.2. The graph has been produced on the basis of data obtained from C. Caldwell as well as from T. Nicely tables of maximal prime gaps. The occurrence of the gap $64 ; \mathfrak{g}=1131$, beginning at the prime 1693182318746371 , visible on the graph at $\xi \approx 10.03$ exhibits the difference $\mathcal{G} s_{(n)}-\mathfrak{g}=0.0132662$.

Theorem 1.3 (Maximal Prime Gaps Supremum and Infimum for primes).

For any $n \in \mathbb{N} \mid n \geq 11$ there exists at least one $p \in \mathbb{N} \mid n<p \leq n+\mathcal{G}_{(n)}=t$; where $p$ is as usual a prime number and the maximal prime gaps upper bound $\mathcal{U B}$ is given by:

$$
\begin{aligned}
& \mathcal{U B}=\mathcal{G} s_{(n)}=5\left(\log _{10} n\right)^{2}-\frac{15}{8}\left(\log _{10} n\right) \quad \forall n \in \mathbb{N} \mid n \geq 11 \\
& \text { Equivalently, } p_{i+1}-p_{i} \leq \mathcal{G} s_{\left(p_{i}\right)}
\end{aligned}
$$




\section{The Nicholson's Conjecture}

John Nicholson [17] in 2013 postulated a conjecture concerning prime numbers distribution and the prime gaps upper bound. His conjecture represents a moderate improvement, as compared to the weak form of the Firoozbakht's conjecture of 1982, please refer to Feliksiak [10].

Theorem 2.1 (Nicholson's Conjecture).

The Nicholson's conjecture is valid for all $p_{n} \in \mathbb{N} \mid p_{n} \geq 11$ :

$$
\left(\frac{p_{(n+1)}}{p_{(n)}}\right)^{n} \leq n \log n
$$

Proof.

Inequality 2.1 is equivalent to:

$$
p_{(n+1)} \leq p_{n} \sqrt[n]{n \log n}
$$

Hence, we obtain the expression for an upper bound on an arbitrary prime gap:

$$
p_{(n+1)}-p_{n} \leq p_{n}(\sqrt[n]{n \log n}-1)
$$

It is obvious, that Nicholson's conjecture worst case scenario, occurs at the Maximal Prime Gaps. If Theorem 2.1 holds at the Maximal Prime Gaps, necessarily, it must hold at every other prime gap. Suppose that the inequality 2.3 is false for $p_{n} \in \mathbb{N} \mid p_{n} \geq 1657$. This implies that:

$$
p_{(n+1)}-p_{n}>p_{n}(\sqrt[n]{n \log n}-1)
$$

Theorem 1.3, specifies the upper bound on maximal prime gaps, therefore, in accordance with the hypothesis, we must have:

$$
\left(p_{n}(\sqrt[n]{n \log n}-1)\right)<\left(5\left(\log _{10} p_{n}\right)^{2}-\frac{15}{8}\left(\log _{10} p_{n}\right)\right)
$$

Cauchy's Root test at $p_{n}=1657$ :

$$
\sqrt[n]{\left|a_{n}\right|}=\sqrt[n]{\left|\left(p_{n}(\sqrt[n]{n \log n}-1)\right)-\left(5\left(\log _{10} p_{n}\right)^{2}-\frac{15}{8}\left(\log _{10} p_{n}\right)\right)\right|}
$$

attains approx. 1.0008404394611654 and asymptotically, strictly from above, tends to 1 , as $p_{n}$ increases unboundedly. Please refer to Figure 2a. By the definition of the Cauchy's Root Test therefore, this implies that the sequence $a_{n}$ diverges. Consequently, since in accordance with the hypothesis:

$$
\left(p_{n}(\sqrt[n]{n \log n}-1)\right)-\left(5\left(\log _{10} p_{n}\right)^{2}-\frac{15}{8}\left(\log _{10} p_{n}\right)\right)<0
$$

the value of inequality 2.7 must decrease as $p_{n}$ increases unboundedly. However, at $p_{n}=1657$ inequality 2.7 attains approx. 1.24411. Since it is a divergent sequence, the difference 2.7 continues to increase as $p_{n}$ increases unboundedly. Please refer 
to Figure 2b. Therefore, we have a contradiction to the initial hypothesis. Hence, necessarily for all $p_{n} \in \mathbb{N} \mid p_{n} \geq 1657$ the inequality is valid:

$$
\left(5\left(\log _{10} p_{n}\right)^{2}-\frac{15}{8}\left(\log _{10} p_{n}\right)\right)<\left(p_{n}(\sqrt[n]{n \log n}-1)\right)
$$

which implies:

$$
p_{(n+1)}-p_{n} \leq p_{n}(\sqrt[n]{n \log n}-1)
$$

For the range of $p_{n} \in \mathbb{N} \mid 11 \leq p_{n} \leq 1657$, direct computer evaluation evidently confirms, that inequality 2.9 holds in this range as well. Please refer to Figure 3. Hence, Theorem 2.1 holds for all $p_{n} \in \mathbb{N} \mid p_{n} \geq 11$. Concluding the proof.
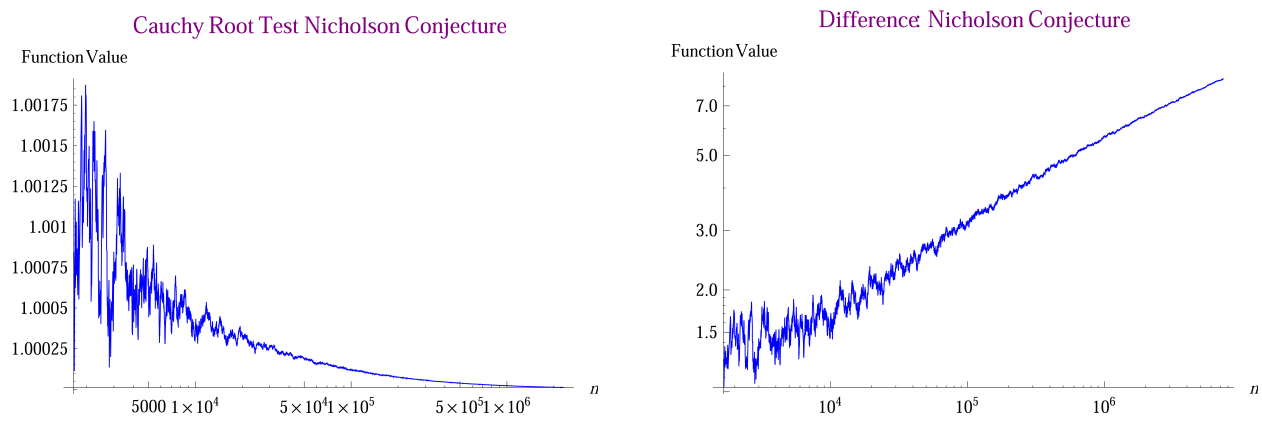

Figure 2. The L.H. log-log drawing shows the graph of the Cauchy root test of 2.6. The figure is drawn over $p_{n} \in \mathbb{N} \mid 11 \leq$ $p_{n} \leq 2307229$. The R.H. log-log figure shows the difference of terms of inequality 2.8 over $p_{n} \in \mathbb{N} \mid 11 \leq p_{n} \leq 7368787$.

Difference: Nicholson Conjecture

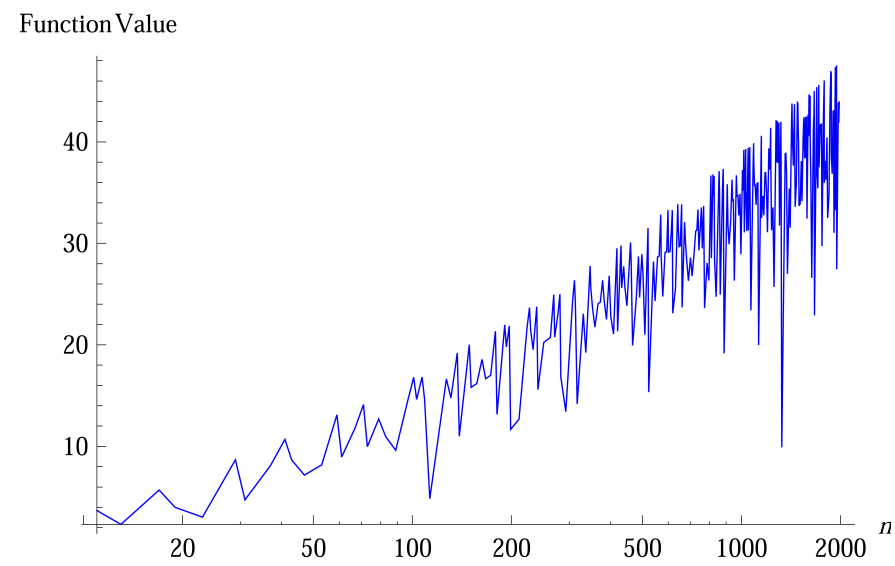

Figure 3 . The drawing shows the graph of the difference of terms of the inequality 2.9 , drawn over $p_{n} \in \mathbb{N} \mid 11 \leq p_{n} \leq 1987$. 
Theorem 2.2 (Nicholson's Conjecture - Supremum Bound).

The Supremum, on the difference of terms of the Nicholson's Conjecture, for all $p_{n} \in \mathbb{N} \mid p_{n} \geq 2$ is given by:

$$
\log n-\frac{1}{n}\left(\frac{p_{(n+1)}}{p_{(n)}}\right)^{n} \leq\left(2\left(\log _{10} n\right)+\gamma^{\frac{\pi}{2}}\right)^{\beta}=\mathcal{S U P}
$$

Where $\beta=\left(\frac{1043}{1000}\right)$ and $\gamma$ is the Euler-Mascheroni constant.

Proof.

Suppose that for $p_{n} \geq 2$ Theorem 2.2 is false. This implies that:

$$
\left(2\left(\log _{10} n\right)+\gamma^{\frac{\pi}{2}}\right)^{\beta}-\left(\log n-\frac{1}{n}\left(\frac{p_{(n+1)}}{p_{(n)}}\right)^{n}\right)<0
$$

However, inequality 2.11 at $p_{n}=2$ attains approx. 1.90643 and asymptotically, strictly from above tends towards 0 as $p_{n}$ increases unboundedly. Please refer to Figure 4a. The Cauchy's Root Test of inequality 2.11, converges asymptotically, strictly from below to 1 . Hence the test is inconclusive. Therefore, to determine the case for convergence/divergence, we implement:

$$
\left.\left[\left(\left(2 \log _{10} n\right)+\gamma^{\frac{\pi}{2}}\right)^{\beta}-\left(\log n-\frac{1}{n}\left(\frac{p_{(n+1)}}{p_{(n)}}\right)^{n}\right)\right)^{(-1)}-p_{n}\right]
$$

At $p_{n}=2$ the difference 2.12 attains approx. -1.47546 and rapidly diverges at a rate $\propto k p_{n}$ with $k \sim 1$, for $p_{n} \in \mathbb{N} \mid p_{n} \geq 2$. Please refer to Figure $4 \mathrm{~b}$. This implies that:

$$
\left(\left(2\left(\log _{10} n\right)+\gamma^{\frac{\pi}{2}}\right)^{\beta}-\left(\log n-\frac{1}{n}\left(\frac{p_{(n+1)}}{p_{(n)}}\right)^{n}\right)\right)>\frac{1}{p_{n}}
$$

Hence, the L.H. of 2.13 tends to zero slower than the R.H. i.e. $1 / p_{n}$. The divergence of the sum:

$$
\sum_{i=1}^{\infty} \frac{1}{p_{i}} \rightarrow \infty
$$

had already been proven by Euler. This implies, that inequality 2.11, converges asymptotically, strictly from above to zero, as $p_{n}$ tends to infinity. Consequently, we have a contradiction to the initial hypothesis. Therefore, for all $p_{n} \geq 2$ Theorem 2.2 holds as stated, concluding the proof.

Corollary 2.3 (Upper bound on the function $\log n$ ).

From Theorem 2.2, the Upper bound on $\log n$ for all $n \in \mathbb{N} \mid n \geq 1$ is given by:

$$
\log n \leq \frac{1}{n}\left(\frac{p_{(n+1)}}{p_{(n)}}\right)^{n}+\left(2\left(\log _{10} n\right)+\gamma^{\frac{\pi}{2}}\right)^{\beta}
$$

Where $\gamma$ is the Euler-Mascheroni constant and $\beta=\left(\frac{1043}{1000}\right)$. 

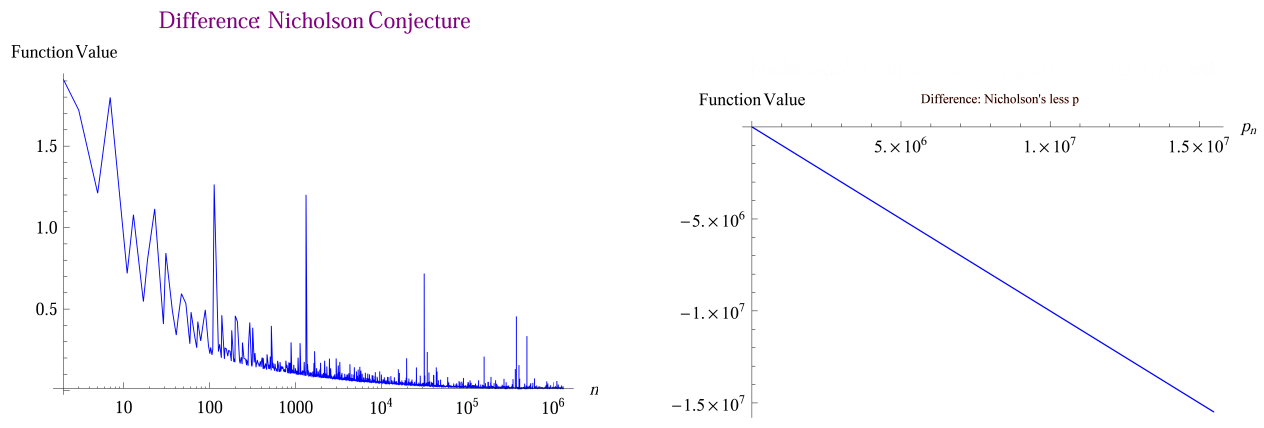

Figure 4 . The L.H. drawing shows the inequality 2.11 , over the range $p_{n} \in \mathbb{N} \mid 2 \leq p_{n} \leq 2307229$. The R.H. drawing shows the difference 2.12 over the range $p_{n} \in \mathbb{N} \mid 2 \leq p_{n} \leq 15485863$.

\section{Supremum bound vs. Nicholson's conjecture}

\section{Function Value}

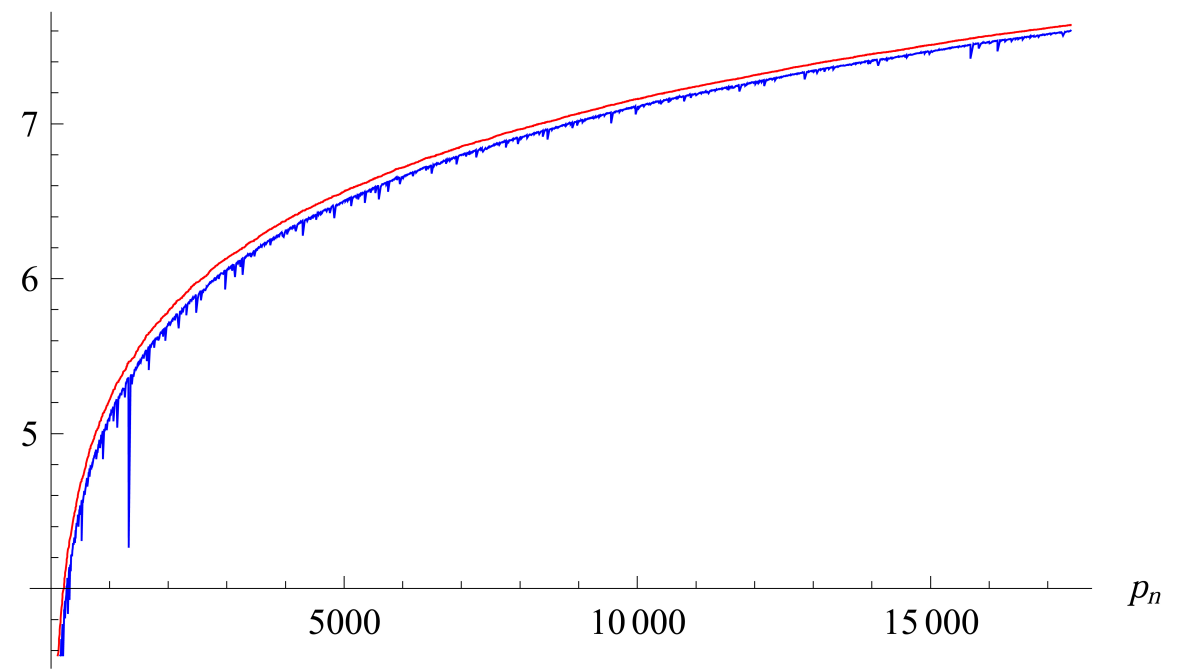

Figure 5. The drawing compares the graphs of the Supremum bound (Red), i.e. R.H. of the inequality 2.10 , vs the difference of terms of Nicholson's i.e. L.H. of the inequality 2.10 (Blue). The figure is drawn over the range $p_{n} \in \mathbb{N} \mid 2 \leq p_{n} \leq 17389$. 


\section{REFERENCES}

[1] R.J. Backlund, Über die Differenzen zwischen den Zahlen, die zu den ersten $n$ Primzahlen teilerfremd sind. Commentationes in honorem Ernesti Leonardi Lindelöf, Annales Acad. Sci. Fenn. 32 (1929), no. 2, 1-9.

[2] A. Brauer and H. Zeitz, Über eine zahlentheoretische Behauptung von Legendre, Sitzungsbericht, Berliner Math. Ges. 29 (1930), 116-125.

[3] Chris K. Caldwell, The gaps between primes, 2006. http://primes.utm.edu/notes/gaps.html.

[4] Harald Cramer, On the order of magnitude of the difference between consecutive prime numbers, Acta Arithmetica (1936).

[5] Paul Erdös, On the difference of consecutive primes, Quarterly Journal Of Mathematics (1935).

[6] Some unsolved problems, Publications Of The Mathematical Institute Of The Hungarian Academy Of Sciences (1961).

[7] _ A survey of problems in combinatorial number theory, Annals Of Discrete Mathematics (1980).

[8] Paul Erdös and E.G. Strauss, Remarks on the differences between consecutive primes, Elem. Math. 35 (1980), 115-118.

[9] Jan Feliksiak, The elementary proof of the Riemann's Hypothesis, MDPI, AG, https://doi.org/10.20944/preprints202006.0365.v1 (2020).

[10] - The maximal prime gaps Supremum and the Firoozbakht's Hypothesis No 30, MDPI, AG, https://doi.org/10.20944/preprints202006.0366.v1 (2020).

[11] _ Maximal prime gaps bounds, ScienceOpen, https://doi.org/10.14293/S21991006.1.SOR-.PPWVKRR.v1 (2021).

[12] _ The Brocard conjecture, ScienceOpen, https://doi.org/10.14293/S2199-1006.1.SOR.PPIH4LV.v1 (2021).

[13] Kevin Ford, Ben Green, Sergei Konyagin, James Maynard, and Terence Tao, Long gaps between primes, arXiv:1412.5029v3[math.NT] (2016).

[14] H Maier, Primes in short intervals, Michigan Mathematical Journal (1985).

[15] Thomas R. Nicely, Some results of computational research in prime numbers (Computational number theory), 2009. http://www.trnicely.net.

[16] _ Skewes' problem, 2009. http://www.trnicely.net.

[17] John Nicholson, Nicholson's conjecture, 2013. https://oeis.org/A182514.

[18] János Pintz, Very large gaps between consecutive primes, Journal Of Number Theory (1997).

[19] Robert A. Rankin, The difference between consecutive prime numbers, Journal London Mathematical Society (1938).

[20] J.B. Rosser and L. Schoenfeld, Sharper bounds for the Chebyshev functions $\theta_{x}$ and $\psi_{x}$, Mathematics of Computation 29 (1975), no. 129, 243-269.

[21] Atle Selberg, On the normal density of primes in small intervals and the difference between consecutive primes, Arch. Mathem. B 47 (1943), 87-105.

[22] Daniel Shanks, On maximal gaps between successive primes, Math. Comp. 18 (1964), 646651.

[23] Tomas Oliveira e Silva, Gaps between consecutive primes, 2006. www.ieeta.pt/ tos/gaps.html.

[24] K. Soundararajan, Small gaps between prime numbers: the work of Goldston-Pintz-Yildirim, Bulletin of the American Mathematical Society (2007).

[25] E. Westzynthius, Über die Differenzen Verteilung der Zahlen die zu den $n$ ersten Primzahlen teilerfremd sind, Comm. Phys. Math. Soc. Sci. Fenn. 5 (1931), 1-37. 